\title{
Underwater Pulse-Current FCAW - Part 1: Waveform and Process Features
}

\author{
Regulating metal transfer using pulse current \\ improved welding process stability
}

BY C. JA, J. WU, Y. HAN, Y. ZHANG, Q. YANG, AND C. WU

\begin{abstract}
The typical metal transfer mode in conventional underwater wet flux cored arc welding (FCAW) delivers large droplet repulsive transfer with low frequency. The process stability and the weld quality are seriously deteriorated with significant spatter and frequent arc extinctions. It is thought the repulsive forces applied on droplets can be reduced by rapidly decreasing the welding current, making the droplets sag and oscillate. A novel underwater pulsecurrent FCAW was proposed to periodically regulate the forces applied on droplets. The experimental system was developed with specially designed pulse current and reliable arc length control. Visual and electrical signals were collected simultaneously to study the process features. It was found that the maximum droplet diameter decreased to less than $5 \mathrm{~mm}$; the temporary arc-extinguishing frequency decreased significantly; there was almost no short-circuit transfer and surface-tension transfer; and the stability of the welding process was significantly improved.
\end{abstract}

\section{KEYWORDS}

- Underwater Wet Welding • Metal Transfer

- Pulse Current • Flux Cored Arc Welding

- Process Control

\section{Introduction}

Underwater welding technology is one of the indispensable technologies in modern offshore engineering, such as offshore platform construction, submarine pipeline laying and maintenance, bridge construction and maintenance, and nuclear power plant maintenance (Refs. 1-4). The selfshielded underwater flux cored arc welding (FCAW) process does not need special drainage equipment and an evacuated chamber for underwater dry welding methods. Compared to manual welding, automated welding has many advantages, including easy operation, high efficiency, and low cost (Ref. 2). Due to the special and complex underwater environment, the quality of underwater wet welding is influenced by many factors, such as arc plasma characteristics, metal transfer, and bubble behaviors. Many studies have proved that metal transfer plays an important role in weld formation, process stability, heat input, and arc burning (Refs. 5-8). Scholars have also conducted a great deal of research on metal transfer control schemes (Refs. 9, 10). Some have reduced the surface tension of droplets by adjusting the type of shielding gas, wire material, and wire feeding methods, thus reducing the size of droplets when they detach from the tip of the wire and decreasing the production of spatter (Refs. 11, 12).

With the performance improvements of the welding power supply (digital, inverters, and controlled waveform), more and more scholars use current waveform to control metal transfer in various welding processes. According to the different characteristics of each phase of metal transfer, corresponding welding current and voltage waveforms were input to ensure a more stable metal transfer and to reduce generated spatter. Among these methods, the most representative one was surface tension metal transfer (Ref. 13). This method is mainly applied in low-current welding. It ensures smooth metal transfer under the effect of surface tension by reducing welding current at the initial stage of short circuit and increasing short-circuit welding current at the later stage of short-circuit transfer. The welding current is significantly reduced at the moment when the droplet contacts the molten pool to form a short circuit; the welding arc is reignited after the metal is transferred to the molten pool, so as to avoid the liquid "bridge" being heated sharply to form a burst resulting in significant spatter. In the process of smooth short-circuit transfer by means of surface tension, the pinch current will be raised again; the electromagnetic contraction force at the neck of the shrunken droplet will be greatly increased, which will have a squeezing effect on the droplet, promoting the metal transfer and shortening the transfer time.

Y. M. Zhang et al. proposed an active control method for pulsed gas metal arc welding (GMAW-P) based on the synergistic use of droplet oscillation momentum and electromagnetic force (Ref. 7). The droplet oscillates when the welding current changes from peak to base. When droplets grow to 


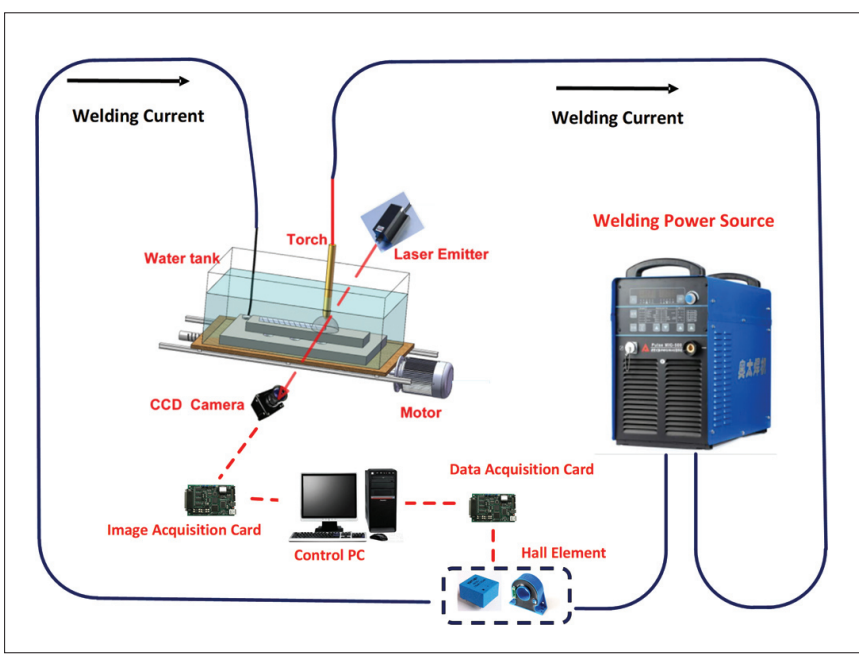

Fig. 1 - Experimental platform and signal acquisition system.

the appropriate size, the pulse current is switched to the valley current, and then the welding current is switched to the excitation current with higher amplitude when droplets oscillate downward. The droplet's downward momentum and the sudden increase of electromagnetic force were used to produce sufficient force to make the droplet detach from the tip of the wire. As a result, the smooth and regulated metal transfer was achieved. In recent years, G. J. Zhang et al. proposed the application of pulsed-laser and negativepulse current to achieve the free transfer of droplets using low welding current (Ref. 14). On one hand, the evaporative reaction force caused by the laser beam was used to promote droplet dropping; on the other hand, the resistant arc force to droplet falling was reduced by rapidly reducing the welding current. The electromagnetic spot resistance of droplets decreased instantaneously with the decrease of the welding current. The droplets changed from a repelled state to a natural sagging state, and returned to the axial position of the wire flexibly (in about $7 \mathrm{~ms}$ ).

The above research provides a theoretical basis for improving the metal transfer in underwater wet FCAW. In conventional wet FCAW, droplets are mainly repelled by complex upward forces in bubbles, which makes it difficult for them to drop freely. They are forced to grow to a large size and even be excluded from the wire axis. According to the analysis of the forces applied on droplets under water (Ref. 15), it is known that the drag force of gas in bubbles mainly comes from the decomposition of flux cores, the decomposition or evaporation of water, and the irregular flow of metal vapor in the molten pool. Therefore, arc heat is the fundamental reason for the repulsive forces. The arc force mainly includes spot pressure, the axial component of electromagnetic force. The repulsive force magnitudes depend mainly on the welding current.

Considering the aformentioned research, merely increasing welding current or voltage can theoretically reduce surface tension and improve plasma flow force, but it will inevitably intensify flux-core decomposition and water decomposition or evaporation, and increase the drag force of gas flow on droplets. At the same time, the arc spot pressure under the droplet increases with the increasing welding cur-

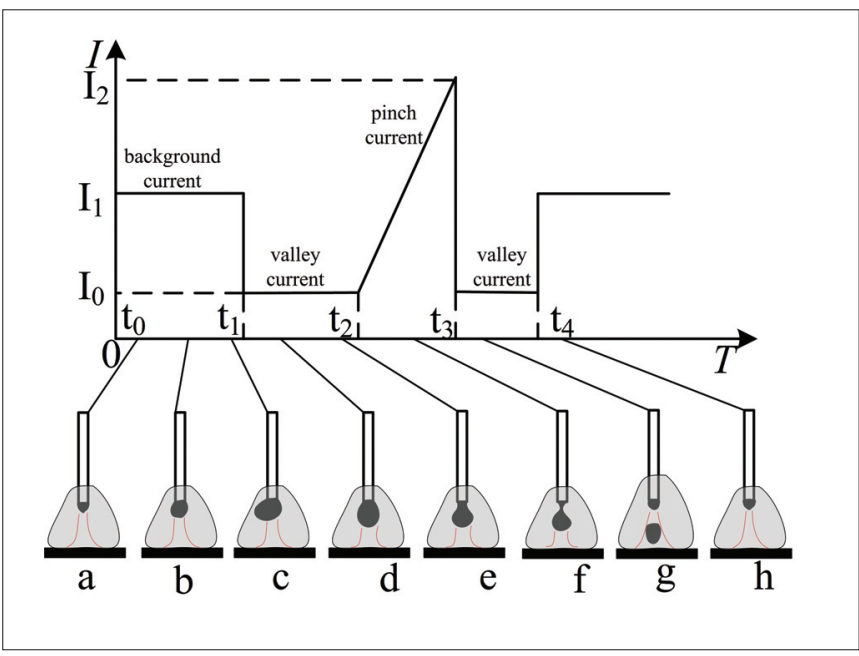

Fig. 2 - Schematic of the pulse-current waveform and assumed metal transfer.

rent. The droplet is still subject to large upward repulsive forces and cannot transfer freely. It is difficult to obtain higher frequency and smaller size metal transfer.

To improve metal transfer stability and weld formation, it was proposed to use pulse current to regulate the metal transfer during underwater wet welding. A novel underwater wet pulse-current FCAW process and its features were investigated. The waveform design, arc length control, and metal transfer processes were comprehensively studied and discussed. The control mechanism, based on pulse current, provided important data and theoretical basis for further optimization of this technology.

\section{Experimental Procedure}

To analyze the relationship between the metal transfer processes and the electrical parameters, a data acquisition and control system was built based on LabVIEW, as shown in Fig. 1. All the experiments were conducted at the water depth of $0.5 \mathrm{~m}$ in a water tank. The data acquisition system included a data acquisition card, terminal board, Hall effect current sensor, voltage sensor, and computer. The sampling acquisition frequency was $100 \mathrm{kHz}$. The range of the voltage sensor was 0 to $100 \mathrm{~V}$. The range of the current sensor was 5 to $500 \mathrm{~A}$. The acquisition and control system can fulfill the requirement of synchronous analog/digital data acquisition and output.

The employed voltage sensor was also designed according to the Hall effect principle. The sensor first converted the collected voltage signals into current form through the designed electric circuit, then output them to the acquisition card. The base metal was Q235 with 8-mm thickness. Beadon-plate welding experiments were carried out using a specially designed flux-cored wire for underwater wet welding made by E. O. Paton Electric Welding Institute, Kiev, Ukraine.

In underwater FCAW, the visual signal acquisition is usually disturbed by many factors, such as bubbles and welding fumes. The fluctuation of the water surface would change 


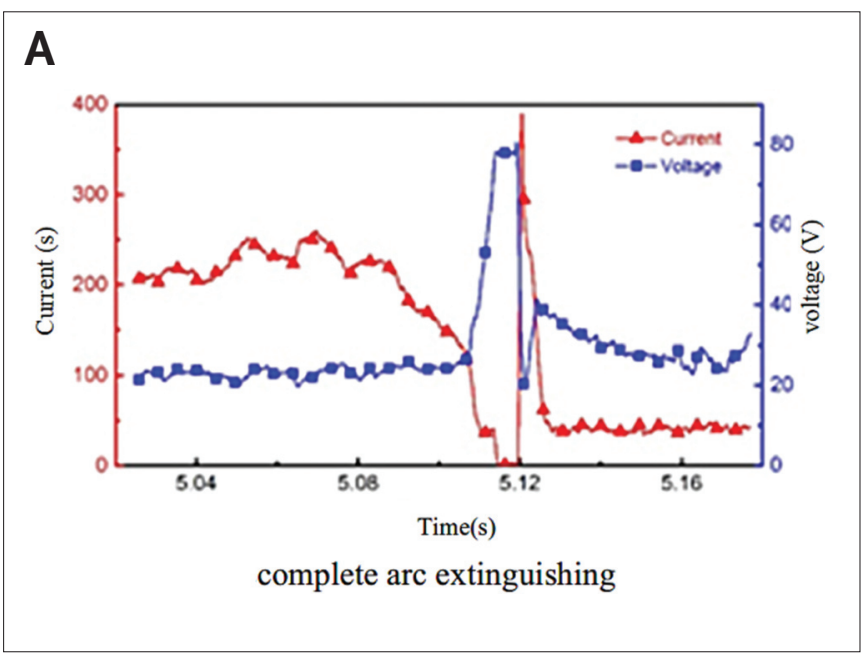

Fig. 3 - Schematic of the closed-loop control system.
B

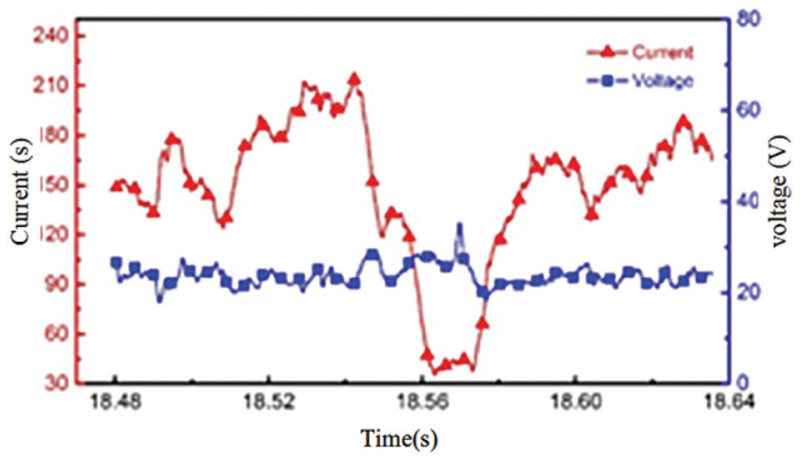

temporary arc distinguishing the optical path from the welding area to the camera. The $\mathrm{x}$-ray-assisted photography technique could obtain metal transfer images, but would lose important information such as the arc and bubble evolution processes (Ref. 16).

In light of the problems detailed above, a visual sensing system based on a high-speed camera was designed. The resolution of the camera was $640 \times 480$ pixels with frame rate up to $2000 \mathrm{fps}$. Clear images of the underwater wet FCAW process (Ref. 17) were obtained, containing rich and important information, including metal transfer, bubble evolution, and arc behaviors. Experiments were designed with the pulse-current waveform, to explore its control effect on metal transfer behaviors in underwater wet welding. Experimental parameters are shown in Table 1 and Table 2. Please note the experiments under constant voltage were conducted to compare and study the welding process stability in pulse-current FCAW.

\section{Results}

\section{Pulse-Current Waveform Design}

GMAW using solid wire, increasing the welding current or voltage can reduce the surface tension and improve the plasma flow force, thus the high-frequency free metal trans- fer or projected transfer can be realized. The free metal transfer mode is the transition form the droplet falls into in the weld pool after falling off from the welding wire end and moving freely through the arc space for a certain distance. However, for underwater wet welding using flux-cored wire, higher current or voltage will inevitably intensify the flux core's decomposition and the water decomposition or evaporation, as well as increase the drag force of gas flow. In addition, the arc spot pressure and electromagnetic force acting upward on the droplets also increase along with the increased welding current. Thus, the repulsive forces are strengthened with a larger extent than the arc forces. The droplets are still subjected to a large upward force and cannot drop freely. As a result, it is difficult to obtain highfrequency droplet transfer with small diameter droplets.

Therefore, the authors proposed that the free metal transfer in underwater wet FCAW can be realized based on pulsecurrent control and regulation. The drag force and arc force were controlled by adjusting welding current, so as to realize the one drop per pulse free metal transfer mode with higher frequency and smaller droplet size. As shown in Fig. 2, the pulse-current waveform was designed with four sections: background current $I_{1}$, valley current $I_{0}$, pinch current $I_{2}$, and valley current $I_{0}$. The preset function of each section was as follows: The waveform of background current is shown in the $t_{0}-t_{1}$ period. The wire melted in a short time at a high current,

Table 1 - Experiment Parameters Under Pulse-Current Mode

\begin{tabular}{|c|c|c|c|c|}
\hline & Wire Feed Rate & Welding Speed & Average Current & Frequency \\
\hline Welding Parameters & $4.4 \mathrm{~m} / \mathrm{min}$ & $180 \mathrm{~mm} / \mathrm{min}$ & $200 \mathrm{~A}$ & $20 \mathrm{~Hz}$ \\
\hline
\end{tabular}

Table 2 - Experiment Parameters Under Constant-Voltage Mode

\begin{tabular}{|c|c|c|c|c|}
\hline & Arc Voltage & Wire Feed Rate & Welding Speed & Wire Diamete \\
\hline Welding Parameters & $27 \mathrm{~V}$ & $4.4 \mathrm{~m} / \mathrm{min}$ & $180 \mathrm{~mm} / \mathrm{min}$ & $1.6 \mathrm{~mm}$ \\
\hline
\end{tabular}




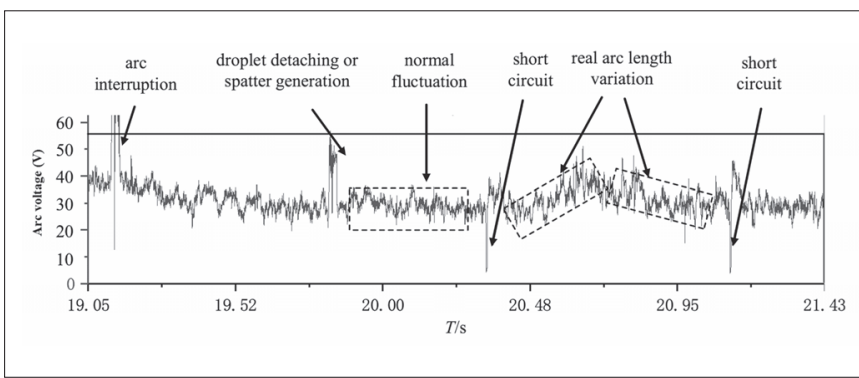

Fig. 4-Arc voltage fluctuation features and recognition in underwater constant-voltage FCAW.

and the droplet grew to a certain size. At that time, there were large repelling forces consisting of the drag force, electric arc force, and buoyancy. At the " $c$ " moment of metal transfer, the grown droplet was repelled by the complex forces, which stopped the droplet's downward movement.

The waveform of valley current $I_{0}$ is shown in the $t_{1}-t_{2}$ period. When the droplet grew to a certain size at $t_{1}$, the welding current was sharply decreased to the valley current. In this case, the repulsive forces decreased subsequently, including the arc force and drag force. The force balance of droplets was then destabilized. According to the previous analysis, it was assumed that the sharply decreased welding current would significantly reduce the repulsive forces. Subsequently the repelled droplet moved downward and hung at the end of the wire. This transition produced a certain oscillation. The droplet size grew larger with the ongoing welding process, and the necking occurred due to the gravity and the downward momentum. The characteristics of metal transfer are shown the moments " $\mathrm{d}$ " and " $\mathrm{e}$ " in the corresponding graphs.

The pinch current $I_{2}$ is shown in the $t_{2}-t_{3}$ period of the figure. Once the droplet grew large enough, the downward forces became higher than the upward forces, as shown at the " $e$ " moment, when the necking phenomenon of the droplet happened. As the welding current was increased sharply from valley current to pinch current, the radial and downward axial components of the electromagnetic contraction forces got much stronger and accelerated the metal transfer process. In addition, the downward inertia momentum offered additional contribution to the droplet detaching process. The detaching and transfer processes are described at moments " $f$ " and " $g$ " in Fig. 2.

Subsequently, the welding current was reduced to valley current $I_{0}$ when the droplet was about to detach from the wire end, so as to prevent the excessive heat input, reduce repulsive forces, and avoid spatter. The characteristics of the metal transfer are shown as " $\mathrm{g}$ " in the corresponding graph. Stable arc burning and bubbles generation were maintained. Then the welding current was raised to the background current to start the next cycle.

\section{Arc Length Control Strategy}

To realize the reliable and accurate output of the preset current waveform, the welding power source was controlled by an external program through an industrial computer. Because the arc self-regulation effect under constant-voltage was not effective in the pulse-current mode, the correspon-
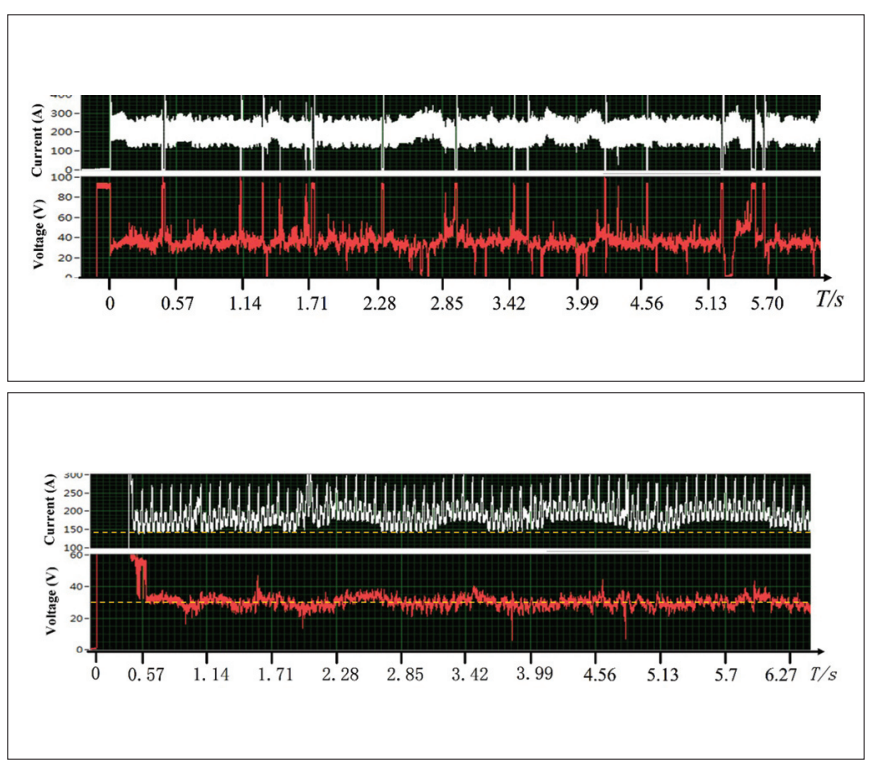

Fig. 5-Collected welding current and arc voltage signals in pulse-current wet FCAW before (top) and after (bottom) applying the arc length control.

ding control for maintaining a stable arc length was critical for FCAW process.

As shown in Fig. 3, a closed-loop control system was developed to avoid arc extinguishing or short circuit caused by arc length variations. The arc length was adjusted by changing the welding current appropriately based on the collected arc voltage signal as feedback. Specifically, when the arc length was too long, the arc voltage was higher than the preset threshold value $(\geq 27 \mathrm{~V})$, the output current of welding power source was reduced, thus the melting rate of the welding wire slowed down, and the arc length was shortened, correspondingly. When the arc length was too short, the arc voltage was lower than the threshold value $(<27 \mathrm{~V})$, the output current was increased to accelerate the melting rate of the wire, and the arc length was then increased accordingly.

Once the difference between the collected and preset threshold voltage was obtained, the controller output a control variable, which decides the average welding current level for the next cycle. As shown in Equation 1, a proportionalintegral (PI) controller was used to calculate the correct output average current in real time. In other words, the amplitude and directions of the welding current adjustment were determined for eliminating the voltage variation.

$$
u_{t}=K_{P}\left[e(t)+\frac{1}{T_{i}} \int_{0}^{t} e(t) d t\right]
$$

where, $u_{t}$ is the output value at $t$ moment, $K_{P}$ is the proportional gain, $T_{i}$ is the integral time, $e(t)$ is the obtained difference between the collected and preset threshold voltages at $t$ moment, and $t$ represents the current moment for calculation. Based on the employed PI controller, the accumulated controlled variables and the initial waveform parameters both determined the required current waveform for a stable arc length. Note that the gain settings associated with the 


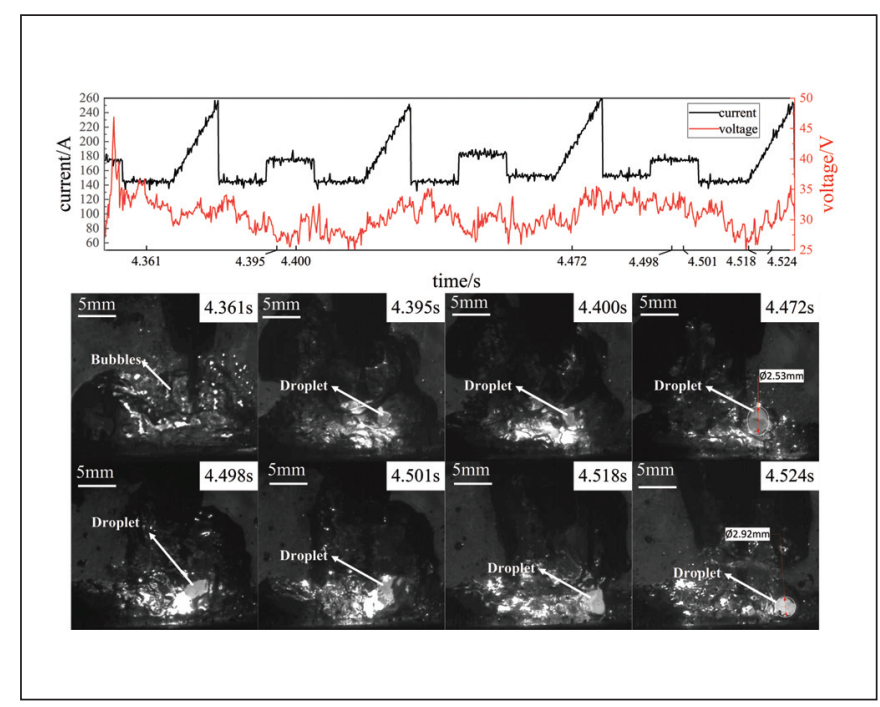

Fig. 6-Typical metal transfer in pulse-current wet FCAW (No. 1).

controller were tuned manually according to experience and experimental data.

However, because the wet welding process is vulnerable to short arcs and frequent disturbances, the above controller might output impropriate values. For example, if arc distinguishing happens, the detected arc voltage would show ultra-high values (close to open-circuit voltage) and the controller would produce an ultra-low welding current; if short circuit happens, ultra-high welding current would be output. Therefore, some restrictions were added to the computer program considering possible exceptions. Thus, the controller became a relatively sophisticated hybrid controller that combined a traditional PI controller with an expert system that is responsible for detecting the disturbances and avoiding wrong output.

Figure 4 shows the arc voltage fluctuations and features in underwater constant-voltage FCAW. The features of different signal fluctuation conditions are recognized, including arc interruption, droplet detaching or spatter generation, normal fluctuation, short circuit, and real arc length variation. In the control program, a function of real-time analysis of the signal data was added to the PI controller and acted as an auxiliary expert system. Based on certain judgment conditions, the changing rates of voltage signals in each pulse cycle were calculated for the automatic feature recognition.

When the arc interruption happened, the arc voltage sharply rose to open-circuit voltage $(80 \mathrm{~V})$. When the large droplet detached from the wire end and formed a spatter or transferred to the weld pool, a sudden rise of the arc voltage was observed. During the stable wet FCAW, the arc voltage fluctuations still existed due to the complicated arc and metal transfer processes in the water environment. It was not suitable to recognize the "normal" fluctuations as "abnormal." In other words, the waveform did not need any adjustment. In comparison, the continuously increasing or decreasing arc voltage signals with certain slopes indicated the real changing arc lengths. The control system needs to output the required adjustment of the welding

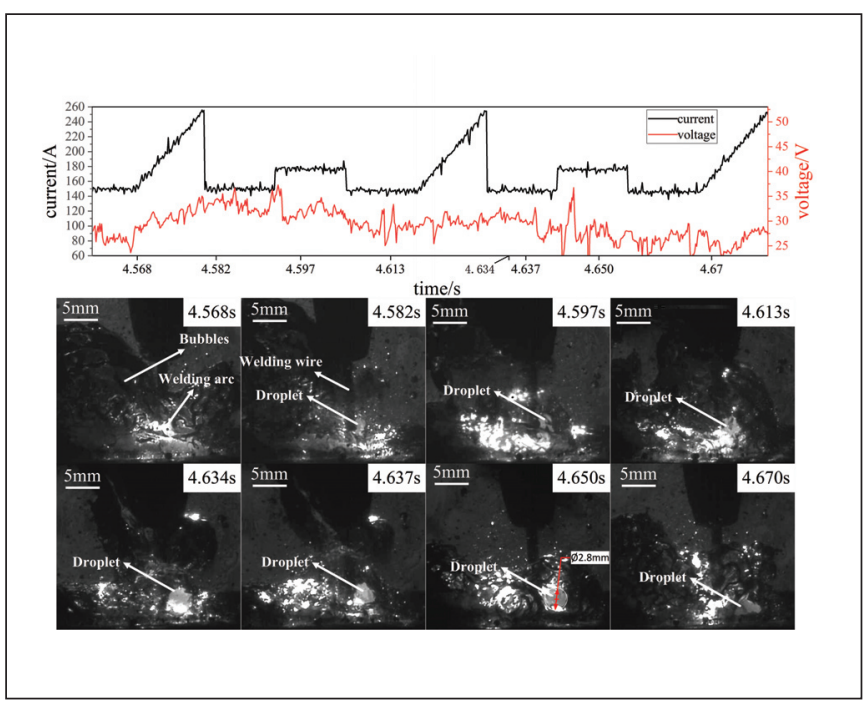

Fig. 7 - Typical metal transfer in pulse-current wet FCAW (No. 2).

current according to Equation 1. In addition, except for the large droplet repulsive transfer, short-circuit transfer happened resulting in the sharply decreased arc voltage (close to 0 ). Considering the above exceptions, the controller output judged the fluctuation types first and gave an appropriate slight adjustment of the current waveform.

Thus, the controller made the right decisions considering the voltage fluctuation trends and other abnormal disturbances. Please note that the update rate of the controller was actually equal to the pulse-current frequency. That is to say, the average current of each cycle of waveform was determined according to the controller output. Additionally, according to the requirement for different welding processes, the pulse and control frequencies ranged from 10 to $100 \mathrm{~Hz}$.

Figure 5 shows the real welding current and arc voltage signals under the pulse-current mode with arc length control. The two sets of signals represented the processes before and after applying the aforementioned arc length control, respectively. It appeared that arc extinguishing and short circuit happened many times during the welding process without arc length control. In comparison, after applying the arc length control strategy, the arc voltage was maintained in a reasonable range; no arc interruption or long-time arc extinguishing happened; the average level of the pulse current was adjusted by the hybrid PI controller. This indicated the well-controlled arc length in the underwater wet FCAW.

\section{Visualization of the Metal Transfer in Pulse- Current FCAW}

Figures 6 and 7 show the synchronously obtained visual and electrical signals in pulse-current underwater wet FCAW. The two selected metal transfer cycles well showed the regulation effect by the pulsed welding current. As shown in Fig. 6, along with the periodically varying welding current, the detailed metal transfer process in a com- 


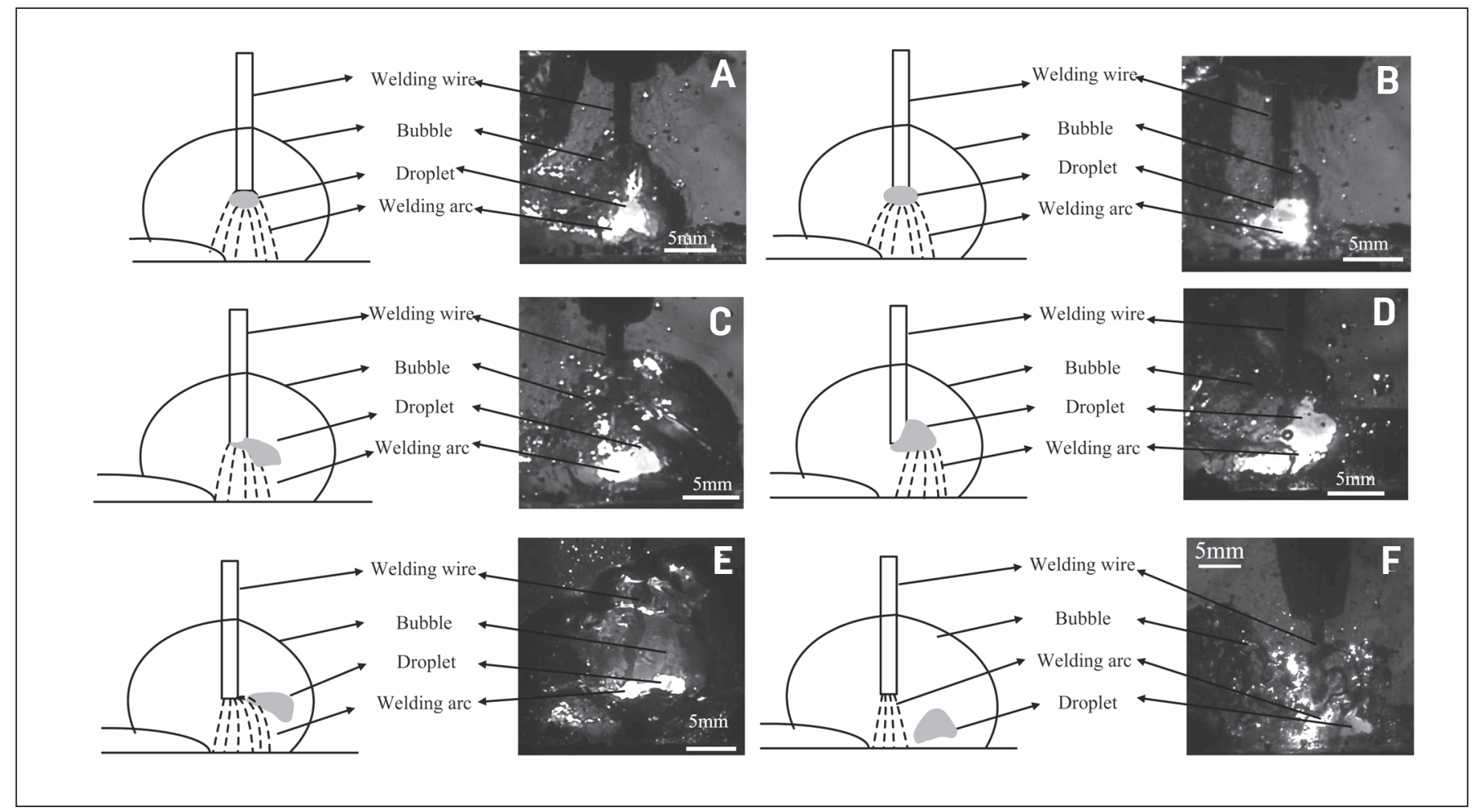

Fig. 8 - Six stages of the typical repelled globular transfer in pulse-current wet FCAW.

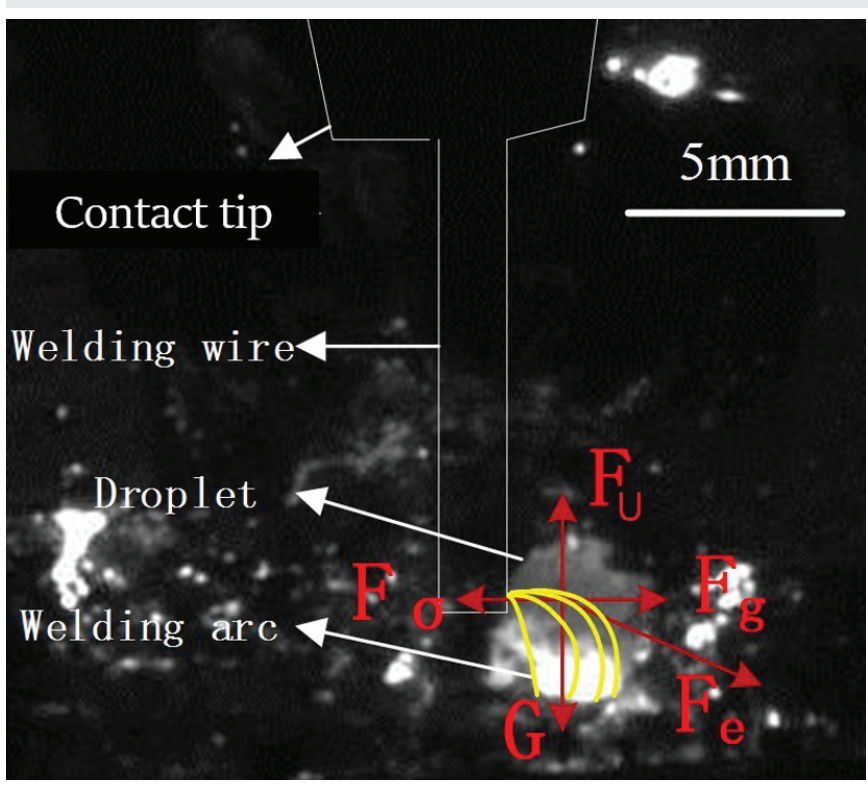

Fig. 9-Force analysis on the droplet at the deviated large droplet stage.

plete cycle from 4.361 to $4.524 \mathrm{~s}$ was clearly observed. Overall, there was only one cycle of metal transfer in three cycles of pulse-current waveform. According to the preset pulsecurrent frequency $(20 \mathrm{~Hz})$, the metal transfer frequency had been increased to $5-6 \mathrm{~Hz}$, which is higher than that in conventional wet FCAW (4.44 Hz) (Ref. 16).

A new liquid droplet was formed at $4.395 \mathrm{~s}$, and the relative positions of the droplet, arc, and wire were clearly seen. Due to the complex situation, the droplet was repelled leav- ing the wire axis with an offset. Subsequently, at $4.472 \mathrm{~s}$ the droplet had grown to an ellipsoid, under the influence from bubbles and welding arc, with a maximum diameter of 2.53 $\mathrm{mm}$ as measured in the figure, which was much smaller than the reported $7.2 \mathrm{~mm}$ in conventional wet FCAW (Ref. 16). In addition, at the moment of $4.472 \mathrm{~s}$, the pinch current was applied by the preset waveform. The droplet was then repelled, due to the pinching forces to the right side at $4.498 \mathrm{~s}$. From 4.498 to $4.501 \mathrm{~s}$, the droplet detached and dropped into the weld pool. It was calculated that the presented metal transfer happened from 4.361 to 4.518 s, which took 157 $\mathrm{ms}$ in total.

Another metal transfer process closely following the finish cycle shown in Fig. 7 was the transfer of the second droplet. The last droplet's detachment time (4.518 s) was set as the starting time of the current metal transfer. A newly formed small droplet was seen at the tip of the wire at 4.582 s. Despite the small volume, the droplet was located on one side of the wire due to the repulsive forces. At $4.613 \mathrm{~s}$, it returned back to below the wire and close to the wire axis. After 119 ms (4.518 to 4.637 s), the droplet grew to a size slightly bigger than the wire diameter. From 4.637 to 4.650 $\mathrm{s}$, the welding current experienced a sudden increase. Accordingly, the arc voltage also increased suddenly and then decreased, and the droplet on the side of the wire detached from the wire end. The droplet maximum diameter was 2.8 $\mathrm{mm}$, and the whole transfer time was $152 \mathrm{~ms}$.

During the two stages, it was noted that the dropletdetaching behaviors of the two droplets were similar. Under the rapidly varying welding current, the droplets were dragged and pushed by complex forces. The oscillation of the liquid droplets was intensified. 

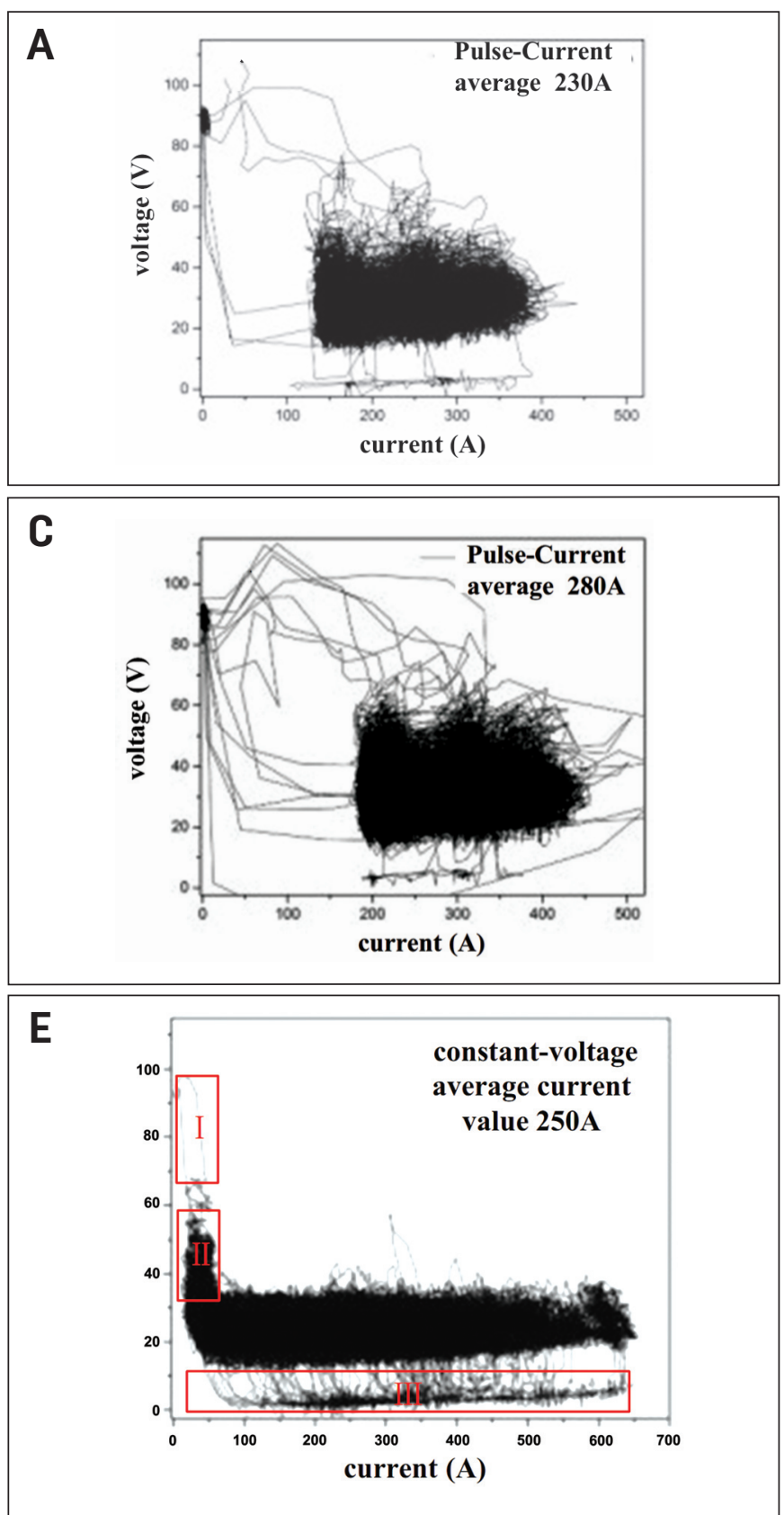
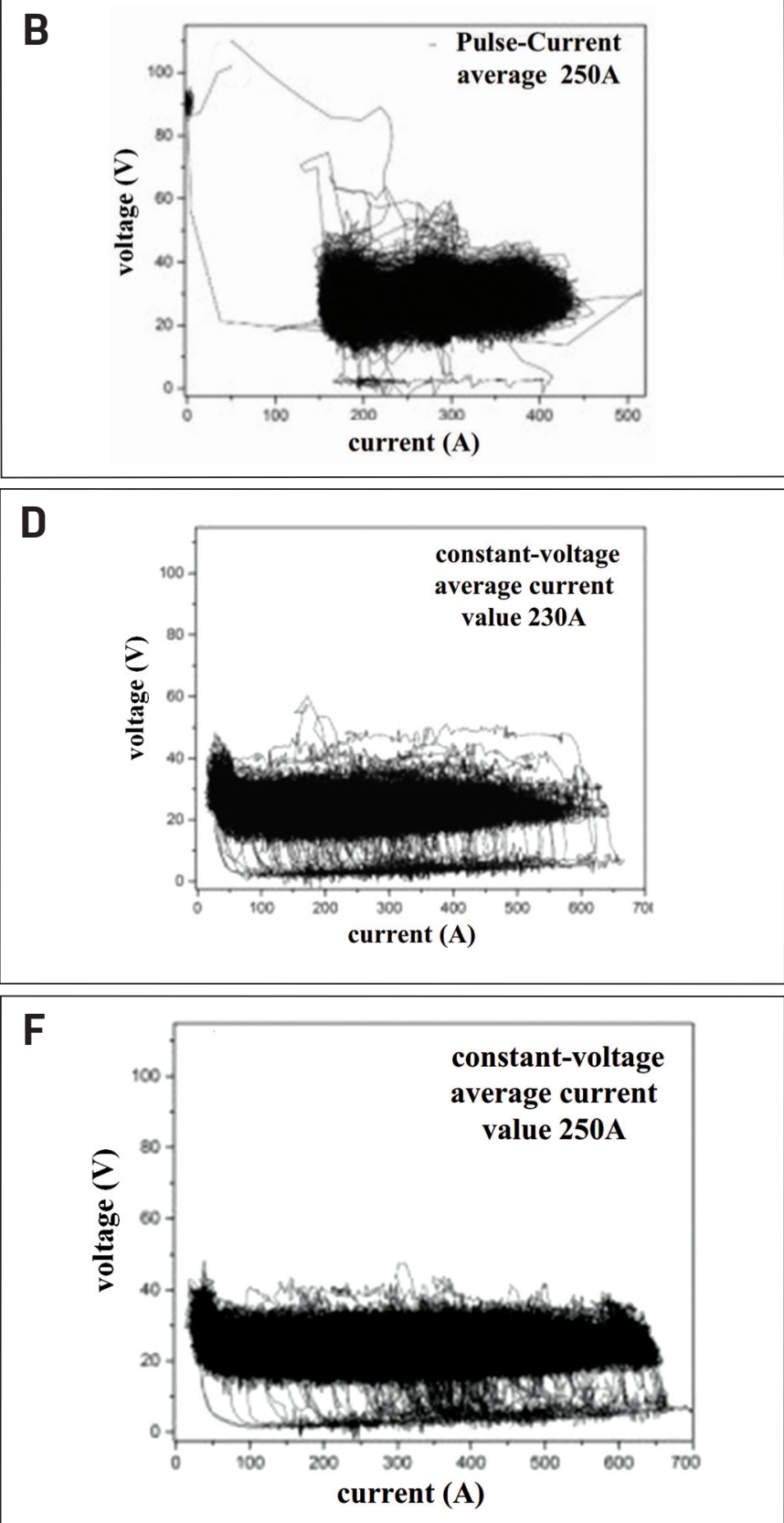

Fig. 10 - Comparison of the U-I diagrams during pulse-current and constant-voltage wet FCAW processes.

\section{Discussion}

\section{Metal Transfer under Pulse Current}

Based on the observation and statistics results, the metal transfer mode in pulse-current wet FCAW is still categorized as repelled globular transfer. However, it had a smaller droplet size and higher frequency than that in conventional wet FCAW. Compared to the proposed control strategy shown in Fig. 2, the real metal transfer seemed different from the assumed situation. The detailed metal transfer process in pulse-current wet FCAW and its characteristics were discussed in this session.
As shown in Fig. 8, a typical metal transfer cycle in pulsecurrent wet FCAW can be divided into six stages according to the relative positions of the arc, droplet, and wire. That is initial stage, growing, deviation, deviated large droplet, detaching, and transferring, as shown in Fig. 8A-F, respectively. It can be seen that the relative positions of the droplet, arc, and wire kept varying along with the increasing liquid volume and changing applied forces.

First, in the initial stage of metal transfer (Fig. 8A), the droplet with small volume hung at the end of the wire and was mostly wrapped in the arc. The resistance forces stopping the droplet from detachment played the predominant role. For example, the surface tension exerted a great influence on the liquid metal. Comparatively, the downward pro- 


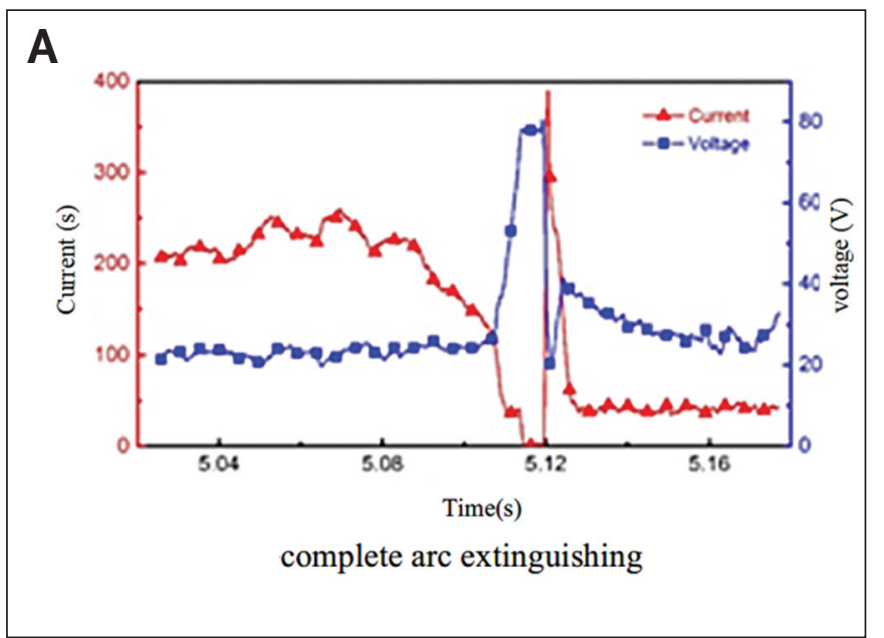

B

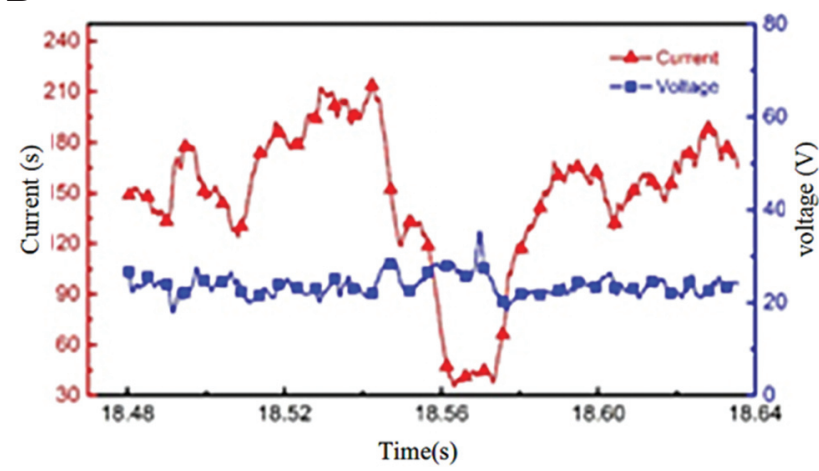

temporary arc extinguishing

Fig. 11 - Electrical signal fluctuations: A - Complete; B - temporary arc extinguishing under the constant-voltage mode.

motion forces applied on the droplets, such as gravity, electromagnetic force, and plasma flow force, were lower.

As shown in Fig. 8B, the droplet continued to grow until its diameter exceeded that of the wire. The welding arc could not cover the droplet volume anymore. The arc anode spot was located on the surface below the liquid droplet. Therefore, the plasma-flow force did not apply to the droplet. Under the impact of gas flow due to thermal decomposition of the flux core, the inner gas flow of the bubbles, and so forth, the repulsive forces pushed the droplet upward and to the side of the wire, as shown in Fig. 8C.

Note that when the FCAW was carried out in air, the grown droplet also deviated to one side of the wire. However, it soon detached from the wire end and was transferred to the molten pool in a short time. Comparatively, in an underwater environment, due to the special interactions between the bubbles, arc, and droplets, the droplet would be pushed upward and outward by the repulsive forces. Thus, the droplet continued to grow when hanging at the wire end and deviated from the wire axis, as shown in Fig. 8D.

When the droplet continued to grow until the moment shown in Fig. 8E, the downward promotion forces, especially gravity and electromagnetic force, prevailed. The droplet was then forced to detach from the wire end and transferred to the weld pool, as shown in Fig. 8F.

It should be noted that the given ideal six stages are not always in accordance with the real situation. For example, during the first two stages, i.e., the initial stage and the growing stage (Fig. 8A and B), the droplet rotated or deviated from the wire rather than keeping coaxial; however, the surface tension force made it return back in a short time. For the mentioned deviation stage (Fig. 8C), the droplet had a sufficient volume, and the surface tension was offset by the increased gravity; the droplet could not return back anymore. Comparing the conventional constant voltage and the pulse-current FCAW processes under the same average current level, the three stages (Fig. 8A-C) occurred over almost the same length of time, approximately 60-70 ms. Therefore, it could be concluded that shortening the time length of the three stages requires higher heat input, which could be considered for following waveform optimization.

For the deviated large droplet stage (Fig. 8D), the time length varied in a large range. In conventional constantvoltage wet FCAW, this stage lasted for 150 to 200 ms. Comparatively, in the pulse-current condition, the time length changed from $30 \mathrm{~ms}$ to $200 \mathrm{~ms}$. That is to say, the applied pulse current in this stage significantly influenced the droplet detaching behaviors. In the situation of the minimum value of $30 \mathrm{~ms}$, the pulse current decreased the metal transfer cycle in a large amplitude. However, the effect of the present pulse current was not stable and accurate. Further optimization of the current waveform was required.

\section{Force Analysis and Pulse-Current Influence}

According to the above analysis, it was found that the long duration of the fourth stage of metal transfer (Fig. 8D), i.e., the deviated large droplet stage, was the main factor causing the long cycle, low frequency, and instability of the metal transfer. It was critical to preliminarily analyze the applied forces on the droplets for precise adjustment and regulation of the behaviors, as shown in Fig. 9.

As can be seen, the droplet was located on one side of the wire, and the welding arc was located right below the droplet. Thus, the droplet and arc were both deviated from the wire axis. The yellow lines represent the main current flow paths. Due to the complex force applications, the liquid droplet was repelled upward and outward. Different forces were discussed based on their orientations.

At this moment, the upward forces $\left(F_{\mathrm{U}}\right)$ applied on the droplet mainly included buoyancy, the drag force, arc forces, and so on. The buoyancy was produced because the droplet was immersed in the bubble gases, which was relevant to its volume and density differences. The drag force was due to the rapidly flowing gas inside the bubbles. The arc forces were mainly composed of anode spot forces and evaporation recoil force. Note that the plasma flow force can be neglected because the large-size droplet was completely outside and above the arc plasma flow. Generally, the resultant upward force was expressed as $F_{\mathrm{U}}$. The downward force was mainly the gravity $(G)$, which increased along with the increasing droplet volume. Please note that the generated electromagnetic pinching force pointed to the welding current paths. Because the droplet was repelled upward and outward, the 

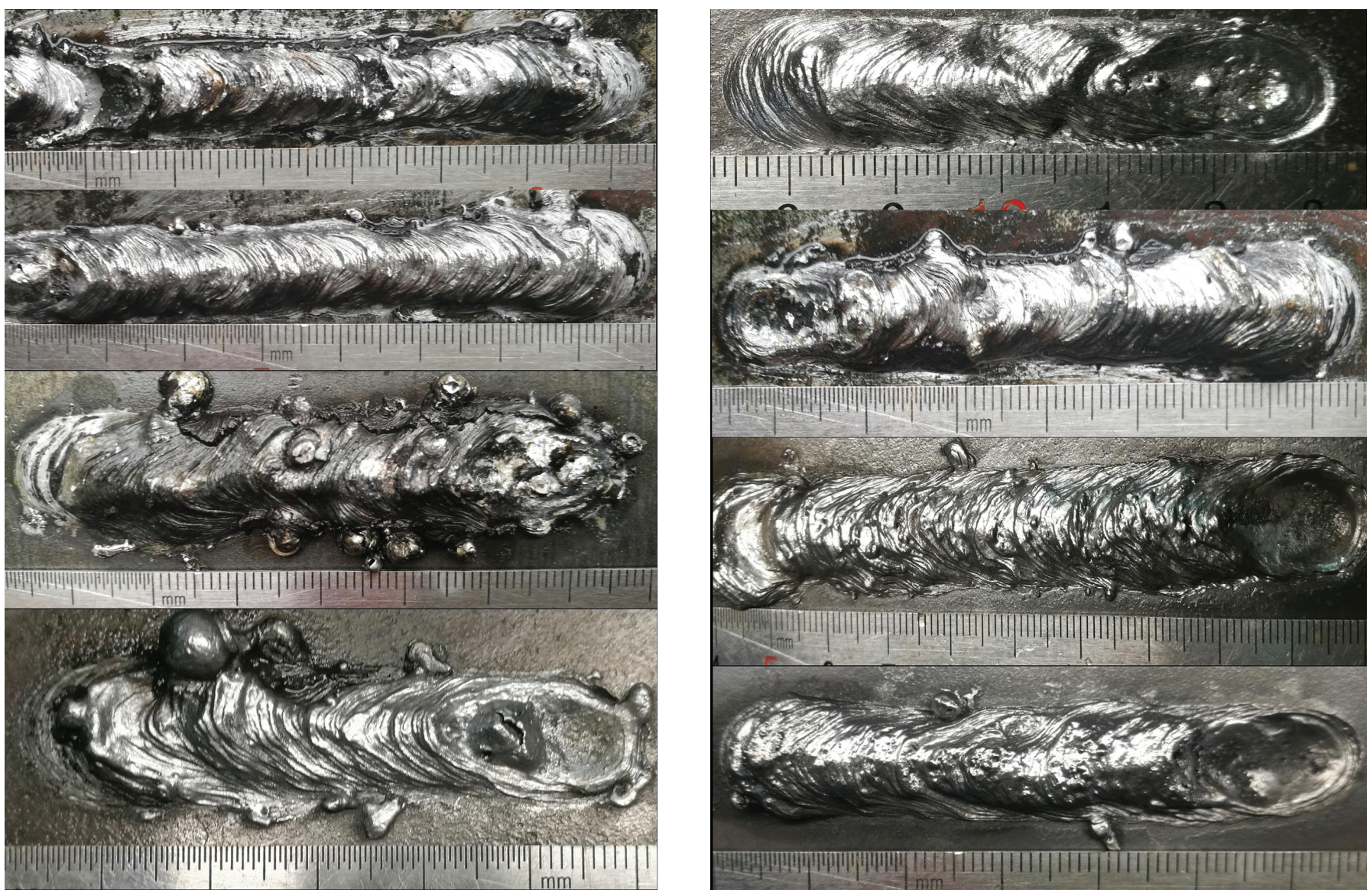

Fig. 12 - Comparison of weld appearance under constant-voltage (left) and pulse-current (right) modes.

dramatically changing shapes at the necking point caused that the resulting pinching force $\left(F_{\mathrm{e}}\right)$ direction was slanted downward and outward.

It was thought the rightward forces (pushing the droplet away from the wire) were mainly caused by the complex and turbulent gas flow (marked as $F_{\mathrm{g}}$ ). On one side, the thermal decomposition of flux core continuously released an amount of gases not only from the droplet but also from the below weld pool. On the other side, the surrounded water was ionized and generated an amount of $\mathrm{H}_{2}, \mathrm{O}_{2}$, and vapor. The surface tension $F_{\sigma}$ tried to pull the droplet back to the wire axis; therefore, its direction was approximately towards the left.

Overall, the force equilibrium on the droplet was maintained temporarily under the above condition. Although the droplet volume continued to increase, it would not detach from the tip of the wire until its diameter was three to four times that of the wire diameter, and the gravity $(G)$ was large enough. The special relative positions delayed the droplet necking and detaching processes; large-size droplets were required due to the gas flow influences in the underwater environment.

Under the condition of pulse current, the droplets were forced to oscillate at this stage (the deviated large droplet stage), as shown in Fig. 7 (note that the oscillation can be more easily recognized from the video). It was thought that the rapidly changing welding current resulted in rapidly changing forces. The force equilibrium was frequently disturbed, which caused the droplet to oscillate. The oscillation kept stretching or shoving the large droplet from time to time. Necking was easier to occur, which further strengthened the electrometric pinching force $\left(F_{\mathrm{e}}\right)$ and reduced the surface tension $\left(F_{\sigma}\right)$. Thus, for the metal transfer, the resistance force decreased and the promoting force increased. As shown in Figs. 6 and 7, at 4.518 and 4.670 s, respectively, the two droplets detached from the wire end and were transferred along the slant downward and outward trajectories.

The experiments showed that the applied pulse current could accelerate the metal transfer process in wet FCAW. However, the duration of the deviated large droplet stage varied in a large range (from 30 to $200 \mathrm{~ms}$ ). It was thought that the pulse current waveform did not match the corresponding metal transfer processes very well. The improvement of reducing the metal transfer cycle and increasing its frequency was not stable.

Therefore, according to the experimental data and analysis, the pulse-current waveform needed to be optimized to change the current immediately when the droplet entered the deviation and deviated large droplet stages. Thus, the relevant forces could be changed, promoting the droplet detachment from the wire as soon as possible. The deviated large droplet stage, as well as the whole metal transfer cycle, could be greatly shortened.

\section{Welding Process Stability}

The U-I diagrams during conventional constant-voltage 
and pulse-current wet FCAW processes were compared to examine the welding process stability as shown in Fig. 10. Experiments were designed using the same level of average welding current. Overall, the U-I distribution in pulsecurrent welding was more concentrated, as shown in Fig. $10 \mathrm{~A}-\mathrm{C}$, than in constant-voltage mode, as shown in Fig. 10D-F. That means that most of the time the electrical signals were stable without violent fluctuations; the pulsecurrent welding process was more stable than the constantvoltage mode. In addition, the proportions of low $(<50 \mathrm{~A})$ or high (> $450 \mathrm{~A}$ ) welding current areas were apparently much lower than that the constant-voltage mode. It was thought that low the welding-current area meant arc extinguishing, or at least unstable arc burning. Therefore, it can be concluded that the arc-extinguishing frequency in the pulsecurrent mode was much lower than that in the constantvoltage mode.

Analyzing the typical U-I signals during constant-voltage wet FCAW in Fig. 10D-F, it was found that the U-I signals were gathered in a larger range than the pulse-current conditions. According to the characteristics, three typical disturbance areas were given in Fig. 10E, including the complete arc-extinguishing region (I), the temporary arc extinguishing region (II), and the short-circuit transfer region (III). The three special U-I areas were used to help analyzing the welding stability differences between constant-voltage and pulse-current wet FCAW. According to the authors' observation, there were mainly two types of arc extinguishing in underwater welding, namely complete arc extinguishing and temporary arc extinguishing. The corresponding electrical signals are shown in Fig. 11.

As shown in Fig. 11A, in the complete arc-extinguishing period, the fluctuation of the electrical signals were typical and similar to conventional GMAW in air. Actually, the arc was completely extinguished; the volume of bubbles decreased sharply; and the continuity of welding process was greatly affected. The characteristics of the temporary arc extinguishing were different because the arc was only temporarily extinguished as seen in Fig. 11B. In the short period, a sharply decreased welding current and a slightly increased arc voltage were observed. The surrounding bubble did not shrink much and continued to shield the arc-burning area. In a short time, the arc was reignited without direct contact between the wire and the weld pool.

Through analysis and comparison, the following characteristics were found. Firstly, the performance of the constant-voltage mode and pulse-current mode in the complete arc-extinguishing area (I) was basically the same. The constant-voltage mode had a slightly less complete arc extinguishing, so this mode was better. Secondly, the proportion of the short-circuit metal transfer area (III) was significantly lower in the pulse-current mode than that in the constantvoltage mode. Previous analysis showed that surface-tension transfer or short-circuit transfer was not a perfect transfer mode for underwater wet welding process. In the process of metal transfer, arc extinguishing and bubble production decreased sharply, which directly affected arc reignition after metal transfer, thus deteriorating the stability of the welding process. In the pulse-current mode, the shortcircuit transfer area took less proportionally, which made the mode of metal transfer more single and improved the metal transfer process. It has been demonstrated that the metal transfer modes are complex (usually two to three kinds) under various parameters in constant-voltage wet welding. The experiments in this paper verified that under the pulse-current mode, the metal transfer mode is single, which was more conducive to enhancing the stability of the underwater wet welding process and to improving the metal transfer mode by other means in the follow-up. Finally, the pulse-current mode performed better in the temporary arc extinguishing area than in the constant-voltage mode. In underwater pulse-current wet welding, there were almost no short-circuit transfer and surface-tension transfer; the frequency of temporary arc-extinguishing was greatly reduced, while the stability of the welding process was improved. Therefore, the performance of the pulse-current wet FCAW was much better than the constant-voltage welding. Frequent arc extinguishing, as well as more spatter and deteriorated process stability, were avoided.

\section{Weld Appearance}

As shown in Fig. 12, the obtained weld appearances under the constant-voltage mode and pulse-current mode were compared. The more stable welding processes resulted in improved weld formation.

The left set of images of Fig. 12 show that the weld appearance varied significantly even with the exact same welding parameters. It indicated that the metal transfer processes, as well as the bubble evolution and arc burning, were not stable. During the complicated welding processes, the weld formation was influenced randomly by the surrounding water without any regulation. Although good topside appearance was occasionally achieved in the second one, more spatter or interrupted weld was observed in the other three experiments, which led to overlap defects on the surface or discontinuous welds.

Comparatively, as shown in the right set of images of Fig. 12 , the obtained welds under the pulse-current mode were generally acceptable. Based on the regulated metal transfer by pulse-current waveform, the generated spatter was significantly reduced, and more uniform welds were acquired. In this case, the pulse current successfully avoided the droplets flying outside the bubbles and changing into spatter.

\section{Conclusions}

1. A novel underwater pulse-current wet FCAW was proposed to regulate complicated metal-transfer processes. The corresponding current waveform was preliminarily proposed, designed, and realized. The arc length control strategy was designed to maintain stable arc burning. Acquired electrical signals showed arc voltage was successfully maintained in a reasonable range; no arc interruption or complete arc extinguishing happened; and arc length control was effectively maintained using a hybrid controller.

2. Analyzing the synchronously collected electrical and visual signals in two typical metal transfer cycles, the metal transfer mode in pulse-current FCAW is still categorized as repelled globular transfer. Compared to the initially proposed control strategy, the regulated metal transfer needs 
three or four pulse cycles to transfer one droplet, which does not match the assumed situation.

3. According to the relative positions between droplet, arc, and wire, the metal transfer process under pulse current can be divided into six stages, i.e., initial stage, growing, deviation, deviated large droplet, detaching, and transferring. The time length for the deviated large droplet stage varied in a large range. The applied pulse current changed the period from 150 to $200 \mathrm{~ms}$ in the constant-voltage mode into 30 to $200 \mathrm{~ms}$. Long duration of the deviated large droplet stage was the main factor causing the long cycle, low frequency, and instability of the metal transfer. The pulse current in this stage significantly influenced the droplet detaching behaviors and decreased the metal transfer cycle in a large amplitude. However, the effect of the present pulse-current was not stable and accurate.

4. Force analysis showed that the special relative position of droplets to the welding wire changed the direction of arc force. The upward forces applied on the droplet mainly included buoyancy, the drag force, and arc force. The rightward forces (pushing the droplet away from the wire) were mainly caused by the complex and disorderly gas flow $\left(F_{\mathrm{g}}\right)$. Special relative positions delayed the droplet necking and detaching processes; large-size droplets were required due to the gas flow influences. The rapidly changing welding current resulted in rapidly changing forces; therefore, the applied pulse current forced the droplets to oscillate at the deviated large-droplet stage.

5. Process stability analysis showed that, in pulse-current wet FCAW, there was almost no short-circuit transfer and surface-tension transfer; the frequency of temporary arc extinguishing was greatly reduced while the stability of welding process was improved. The performance of the pulsecurrent wet FCAW was much better than that of constantvoltage welding, avoiding frequent arc extinguishing, spatter, and deterioration of the process stability.

6. During the constant-voltage welding process, the weld formation was influenced randomly by the surrounding water without any regulation, causing spatter or an interrupted weld. Comparatively, the pulse-current FCAW obtained optimized weld formation by regulating the metal transfer and avoiding the droplets flying outside the bubbles and changing into spatter. Additionally, the effect of the underwater bubbles cannot be neglected and the behavior of the bubbles under pulsed current needs further analysis.

\section{Acknowledgments}

The authors are grateful to the National Natural Science Foundation of China (Grant No. 51675310) and the Fundamental Research Funds of Shandong University (No. 2015TB002) for their financial support of this project.

\section{References}

1. Perez-Guerrero, F., and Liu, S. 2005. Maintenance and repair welding in the open sea. Welding Journal 84(11): 54-s to 59-s.

2. Rowe, M., and Liu, S. 2001. Recent developments in underwater wet welding. Science and Technology of Welding \& Joining 6(6):

\section{7-396. DOI: 10.1179/stw.2001.6.6.387}

3. Paton, B. E. 1997. PWI activities in the field of underwater welding and cutting technologies. Underwater Wet Welding and Cutting, pp. 1-5. Woodhead Publishing Ltd., Cambridge, England. DOI: $10.1533 / 9780857093165.1$

4. Shi, Y. H., and Zheng, Z. P. 2013. Sensitivity model for prediction of bead geometry in underwater wet flux cored arc welding. Transactions of Nonferrous Metals Society of China 23(7): 1977-1984. DOI: 10.1016/s1003-6326(13)62686-2

5. Han, G. M., Yun, S. H., Cao, X. H., et al. 2003. Acquisition and pattern recognition of spectrum information of welding metal transfer. Materials and Design 24(8): 699-703. DOI: 10.1016/s0261-3069(03)00092-x

6. Haidar, J., and Lowke, J. J. 1996. Predictions of metal droplet formation in arc welding. Journal of Physics D Applied Physics 29(12): 2951-2960. DOI: 10.1088/0022-3727/29/12/003

7. Zhang, Y. M., Liguo, E., and Kovacevic, R. 1998. Active metal transfer control by monitoring excited droplet oscillation. Welding Journal 77(9): 388-s to 395-s. DOI: 10.1007/BF02675825

8. Weglowski, S., Huang, Y., and Zhang, Y. M. 2008. An investigation of metal transfer process in GMAW. Engineering Transactions 56(4): 345-362. DOI: 10.1117/12.846716

9. Tanaka, M., Tashiro, S., Ushio, M., et al. 2006. $\mathrm{CO}_{2}$-shielded arc as a high-intensity heat source. Vacuum 80 (11-12): 1195-1198. DOI: 10.1016/j.vacuum.2006.01.047

10. Meyer, D. W., and Nemchinsky, V. A. 2010. Method of metal transfer regulation during GMA welding. The European Physical Journal: Applied Physics 50(1): 11001. DOI: 10.1051/epjap/2010012

11. Tanaka, M., Tamaki, T., Tashiro, S., et al. 2008. Characteristics of ionized gas metal arc processing. Surface \& Coatings Technology 202(22-23): 5251-5254. DOI: 10.1016/j.surfcoat.2008.06.022

12. Soderstrom, E.J., and Mendez, P. F. 2008. Metal transfer during GMAW with thin electrodes and $\mathrm{Ar}-\mathrm{CO}_{2}$ shielding gas mixtures. Welding Journal 87(5): 124-s to 133-s. DOI: 10.1179/174591908X304153

13. Stava, E. K. 1993. The surface-tension-transfer power source - A new, low-spatter arc-welding machine. Welding Journal 72(1): 25-29.

14. Xiao, J., Zhang, G., Chen, S., and Zhang, H. 2017. Decoupling control of metal transfer in GMAW by pulsed laser irradiation. Transactions of the China Welding Institution 38(6): 33-36.

15. Guo, N., Du, Y., Feng, J., et al. 2015. Study of underwater wet welding stability using an X-ray transmission method. Journal of Materials Processing Technology 225: 133-138. DOI: 10.1016/j.jmatprotec.2015.06.003

16. Guo, N., Xu, C. S., Guo, W., Du, Y. P., and Feng, J. C. 2015. Characterization of spatter in underwater wet welding by $\mathrm{x}$-ray transmission method. Materials \& Design 85: 156-161. DOI: 10.1016/j.matdes.2015.06.152

17. Jia, C. B., Zhang, Y., Zhao, B., et al. 2016. Visual sensing of the physical process during underwater wet FCAW. Welding Journal 95(6): 202-s to 209-s.
CHUANBAO JIA (jiachuanbao@sdu.edu.cn), JUNFEI WU, YANFEI HAN, YONG ZHANG, QINGYUAN YANG, and CHUANSONG WU are with the Ministry of Education Key Laboratory for Liquid-Solid Structural Evolution and Processing of Materials, and the Institute of Materials Joining, Shandong University, Jinan, China. 